\title{
EL ESPÍRITU EMPRENDEDOR: CONDICIONANTES PARA LA INNOVACIÓN
}

\author{
E. LEITE ${ }^{1 *}$, E. B. CORREIA ${ }^{1}$, M. D. SÁNCHEZ-FERNÁNDEZ ${ }^{2}$ e E. LEITE ${ }^{1}$ \\ ${ }^{1}$ Universidade de Pernambuco \\ ${ }^{2}$ Universidade da Coruña \\ emanueleite@hotmail.com*
}

Article recibido en marzo/2015 y aceptado en agosto/2015

DOI: $10.15628 /$ holos.2015.2896

\section{RESUMO}

El propósito de esta investigación es estudiar el fenómeno de los condicionantes de la relación de la innovación y el desarrollo del espíritu emprendedor. Uno de los pilares básicos del desarrollo del espíritu emprendedor es la educación, fuente innovadora inagotable de mejora e innovación. La economía del siglo XXI está orientada al aprendizaje. La promoción del emprendedorismo envuelve un cambio cultural basado en la premisa de que las personas pueden mejorar su calidad de vida y de otros, tomando para sí mismos la tarea de dirigir y definir su propio destino y construyendo los suyos propios. No se trata simplemente de producir más con menos costes, lo que se pretende, principalmente, es fomentar el proceso innovador de la manera más inteligente posible. En este contexto, las universidades adquieren una importancia vital. Ante esto, el espacio ibero-americano debe ser el escenario de colaboraciones para acciones conjuntas de la capacidad de generación de conocimiento ya existente, constituye la dimensión crucial de cualquier esfuerzo de desarrollo en países como Brasil. Hoy en día la participación de la microempresa es clave para el desarrollo de cualquier economía. Así como la actitud emprendedora se contempla como la puerta de entrada para la creación de empresas.

PALABRAS-CLAVE: emprendimiento, microempresa, incubación de empresas.

\section{ENTREPRENEURIAL SPIRIT: DETERMINANTS FOR INNOVATION}

\begin{abstract}
The purpose of this investigation is to study the phenomenon of the determining factors of the relationship between innovation and the development of the entrepreneurial spirit. One of the basic pillars of the development of the entrepreneurial spirit is education; a source of improvement and innovation. The economy of the 21 st century is a learning-oriented economy. The promotion of entrepreneurship involves a cultural change based on the premise that people can improve their quality of life and of others by directing, defining and creating their own destiny. It is not simply about
\end{abstract}

producing more at a lower cost, what it is mainly intended is to foster innovation process in the most intelligent manner. In this context, universities take on vital importance. Before this, the Ibero-American space should create partnerships and joint actions for the capacity of the generation with existing knowledge. This constitutes the crucial dimension of any effort of development in countries such as Brazil. Nowadays the participation of micro-enterprises is the key for the development of any economy. Entrepreneurial attitude is also seen as the gateway to the creation of companies.

KEYWORDS: entrepreneurship, microenterprise, incubation. 


\section{INTRODUCCIÓN}

En el presente trabajo analizaremos cuatro temas vinculados a la actitud emprendedora, llamada también emprendimiento, espíritu emprendedor y en inglés entrepreneurship. Este trabajo busca diseminar las condiciones que posibilitan el desarrollo de las empresas y el emprendimiento, más particularmente, la creación de nuevas empresas, mediante incubadoras, como fuente innovadora.

En primer lugar mostramos una visión general de la economía en la sociedad del siglo XXI para mostrar el contexto actual y lo que nos depara en el futuro. Seguidamente nos referimos a la microempresa, cuya presencia es una de las características de la economía de este siglo XXI y guarda relación, obviamente, con la apertura de oportunidades para los emprendedores. La creación de una empresa de este es, normalmente, el primer paso de muchos emprendedores.

En el segundo bloque abarcamos las fuerzas motrices o dinamizadoras de la economía, para su desarrollo nos apoyaremos en algunas investigaciones recientes, después abordaremos la temática del emprendimiento.

Finalmente analizamos como la innovación puede contribuir de forma eficiente y eficaz en el desarrollo del emprendimiento, la incubación y el start-up.

\section{REVISIÓN DE LA LITERATURA}

\subsection{La economía del siglo XXI}

\subsubsection{La importancia de la educación}

En primer lugar desarrollaremos las tendencias y las características de la economía del siglo XXI. Reflejamos seguidamente una breve síntesis de las principales ideas sobre esta visión: a) el paso de la sociedad industrial a la sociedad de la información; b) de la economía nacional cerrada a la economía mundial - estamos cada vez más en una economía más globalizada; es difícil pensar en los próximos veinte años, porque siempre los problemas se presentan hoy y parece que estamos encerrados en el corto plazo; c) de la centralización a la descentralización d) de la ayuda estatal o del estatismo a la auto-ayuda, la auto-afirmación; e) de la democracia representativa a la democracia participativa; $y$ f) de las jerarquías a las redes.

También hacemos referencia a un texto de gran transcendencia que diseña brillantemente la sociedad del futuro, se trata de la sociedad post-capitalista.

El recurso económico básico ya no es ni el capital ni los recursos naturales, ni el trabajo, sino que es y seguirá siendo el conocimiento (LEITE, 2012). El capital y el trabajo, que fueran fuentes creadoras de riquezas durante los siglos XIX y XX y que están presentes en la Teoría Económica Clásica, Marxista, Keynesiana y Neoclásica, perdieron vigencia desde el siglo XXI. Los valores son creados ahora por la productividad y por la innovación, ambas aplicaciones del conocimiento al trabajo. No dejando de ser necesario el capital el conocimiento se convierte en la fuente principal del emprendimiento, pues el emprendimiento y el conocimiento dan lugar a la creación de riquezas. 
Se puede observar que la educación es una gran plataforma transformadora de la sociedad y que toda mejora significativa a nivel social, nacional, regional o individual será fruto de la educación (LEITE, 2012). La educación es inagotable, siempre estaremos mejorándola y ampliando nuestros conocimientos.

\subsubsection{Los Clústeres}

Por otra parte cabe señalar la importancia de los clústeres, lo que conocemos como las concentraciones geográficas de empresas e instituciones de éxito competitivo en un determinado campo o actividad.

El mapa económico mundial está dominado por clústeres. La solidez de las ventajas competitivas de una economía global se basa cada vez más en aspectos locales, ya sea en conocimientos, relaciones y motivaciones que los rivales distantes no pueden igualar. Como ejemplos de clúster, encontramos entre los más tradicionales el entretenimiento en Hollywood, las finanzas en Wall Street y la electrónica de consumo en Japón. Otros ejemplos de gran importancia exportadores en los EEUU son: el clúster vinícola en California, el aeronáutico en Seattle, la petroquímica en Texas, la biotecnología en Boston, los semiconductores en Phoenix, la microelectrónica y el capital de riesgo en Silicon Valley, California. También podemos encontrar ejemplos en Brasil como el del municipio de Petrolina en el Estado de Pernambuco (LEITE, 2012).

Las condiciones de análisis de la realidad capitalista en el espacio innovador se cimientan en aspectos estructurados sobre la cuestión de la producción, la división territorial del trabajo, los flujos y el carácter informativo (SANTOS, 1994, 1996). La realidad del espacio innovador conduce a una visión en una nueva dinámica socio-espacial, con base en la globalización y en la innovación, cuya búsqueda se ve limitada por la información, la comunicación, el flujo y otros aspectos tales como la nueva mirada geográfica basada en la "proximidad geográfica" (MORGAN, 2004) y el funcionamiento en el que se apoya la ubicación como un impulso al capital de la realidad de la geografía regional y global (CORREIA, 2012).

Los clúster están asociados a la especialización, la concentración de actividades y la interrelación de empresas, de muchas compañías, en espacios geográficos relativamente pequeños, en la búsqueda incesante de la innovación, como podemos contrastar en la figura 1.

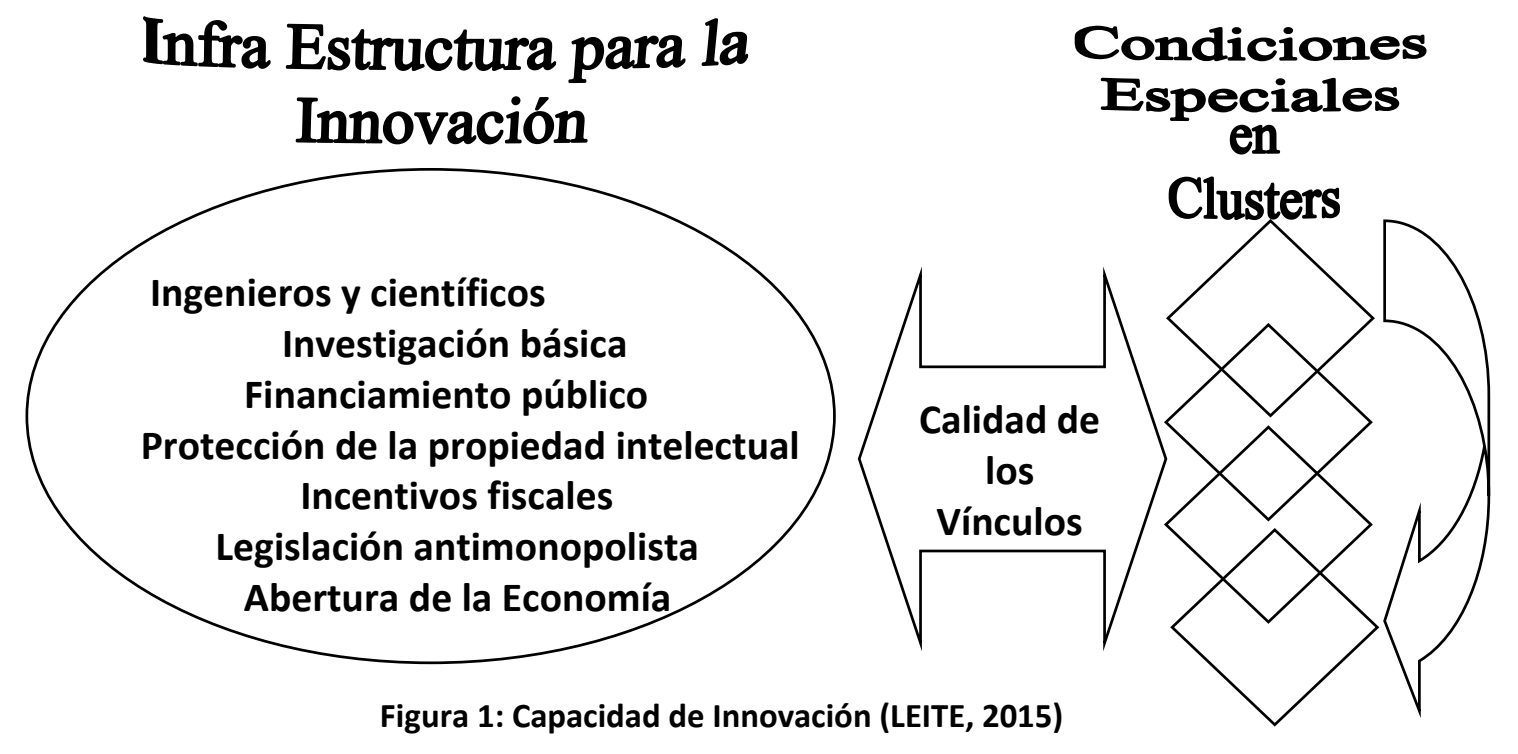




\subsubsection{Enfoque de la Nueva Teoría del Conocimiento}

Iniciamos este epígrafe resaltando el concepto de "especialización flexible" (LEITE, 2015) como el modelo de la organización empresarial del futuro - que, obviamente, va más allá de las empresas - y que se orienta a una visión de la empresa desde la economía del siglo XXI. Por lo que nos preguntamos ¿Qué es lo que caracteriza la especialización flexible? Este modelo de organización empresarial se caracteriza por: la innovación y el dinamismo tecnológico, la reducción de la escala de producción (fenómeno que se observa con claridad desde la década de los 80), la organización horizontal, el rol fundamental y activo de los recursos humanos, la personalización de los productos, el uso intensivo de la información y la cooperación entre empresas e instituciones (principalmente educativas).

Podemos definir la sociedad del siglo XXI como "una economía basada en el conocimiento" (DRUCKER, 1985) que va más allá, "dirigida por la innovación". En esta economía, la clave es la acumulación del conocimiento. Complementando esta síntesis, en un estudio más reciente (LEITE, 1999) se resalta que, la Unión Europea prefiere usar, en lugar de "sociedad del conocimiento", el término "sociedad del aprendizaje" o "economía del aprendizaje", ya que permanentemente debemos aprender cosas nuevas y desaprender otras, haciendo nuevas combinaciones e integraciones, como podemos visualizar en la figura 2.

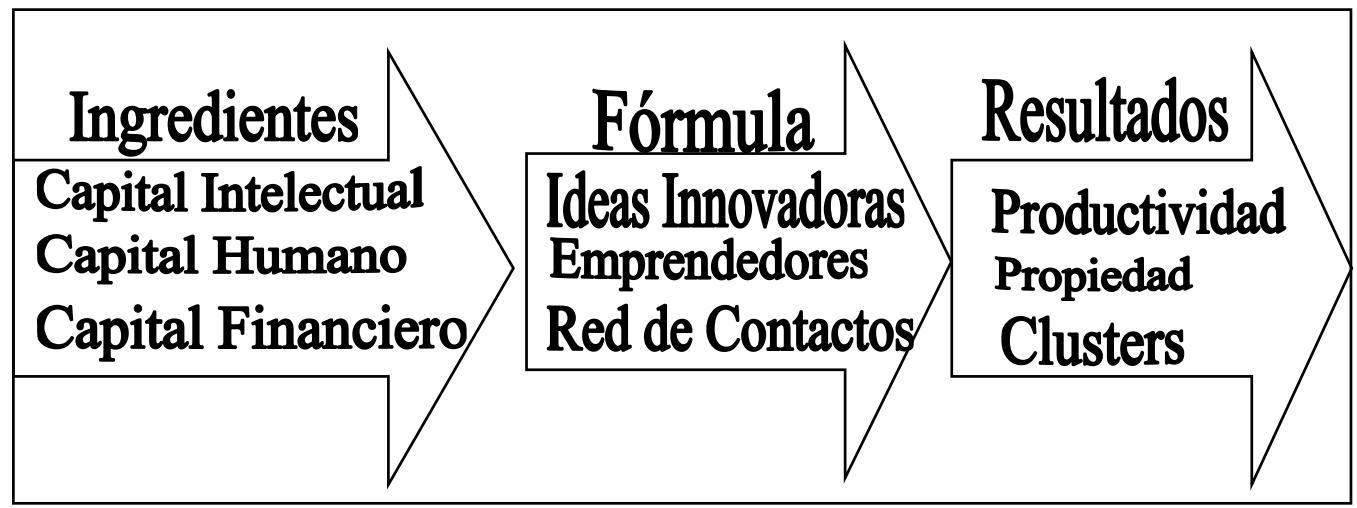

Figura 2: Nueva Teoría del Conocimiento (Fuente: Leite, 2015)

\subsection{El saber contribuye a la competitividad}

Partimos de la premisa de que el principal insumo para el crecimiento económico en los próximos años será el conocimiento - conocimiento especializado y de alto nivel (LEITE, 1999). El punto central es que el conocimiento se transformó en un pilar fundamental de la agregación de valor y la producción de riqueza en el mundo contemporáneo.

La transformación a la cual nos estamos refiriendo confiere centralidad a la Ciencia y a la Tecnología en cualquier esfuerzo de desarrollo, porque de forma más intensa el desarrollo iberoamericano se sustenta en la capacidad de generación de conocimientos. La distribución desigual de la capacidad de creación de conocimiento en el mundo se transformó en la actualidad en uno de los más importantes, si no el principal, instrumento de generación y ampliación de riquezas. Por lo tanto, el espacio ibero-americano no puede ser víctima de una especie de apartheid tecnológico.

Consecuentemente todo ello hace que los países compitan por la atracción del talento y la creación del mismo (SARAIVA e PAIVA). No se trata simplemente de producir más con menos 
costes (tarea en que los asiáticos en la actualidad parecen imbatibles), lo que se pretende, principalmente, es fomentar el proceso innovador de la manera más inteligente posible.

En este contexto, las universidades adquieren una importancia vital. Por lo tanto, los países que se muestran capaces de mantener las mejores universidades tendrán garantizado un lugar prominentemente en el competitivo mundo globalizado. Además de las prácticas de enseñanza, las universidades contribuyen de forma notable en el desarrollo de actitudes emprendedoras (SARAIVA e PAIVA, 2014) Ante esto, el espacio ibero-americano debe ser el escenario de colaboraciones para acciones conjuntas de la capacidad de generación de conocimiento ya existente, consecuentemente constituye una dimensión crucial en países como Brasil.

\subsection{La microempresa}

Prevemos que, en la era del conocimiento, la microempresa, se convertirá en una pieza fundamental en el proceso de creación de empleo y renta. Hoy en día la participación de la microempresa es considerada como un pilar fundamental para el desarrollo de cualquier economía. Así como la actitud emprendedora es considerada la puerta de entrada para la creación de empresas (LEITE, 2012; SARAIVA e PAIVA, 2014). Remontándonos históricamente podemos vislumbrar que la mayoría, si no todas, las empresas han iniciado su actividad como microempresas.

Desde la empresa Ford, a principios del siglo pasado, hasta Microsoft, en la década de los 70, ambas comenzaron en los garajes de las casas de sus promotores y se han transformado en empresas líderes ampliamente reconocidas. Podemos encontrar miles de ejemplos de empresas que iniciaron su actividad como microempresas y hoy en día son compañías gigantes. En el análisis que plasmamos hacemos referencia al modelo fabril aplicado desde la revolución industrial, el cual luego se denominó modelo fordista-taylorista y que fue llevado al extremo, particularmente, por los países socialistas y la Ex Unión Soviética, en donde todo era de grandes dimensiones, estatal y rígido.

Este modelo sirvió para alcanzar una industrialización forzosa para salvar etapas. En los años veinte, cuando fue implementada la Revolución Rusa, la Ex Unión Soviética era una nación muy atrasada, en la Segunda Guerra Mundial ya se había convertido en una potencia industrial militar y política. Esta capacidad industrial fue una de las razones por las cuales se pudo vencer, junto con los Aliados, a la Alemania nazista.

Durante toda esa época y hasta aproximadamente los años sesenta y setenta el modelo funcionó; luego comenzó a estancarse, cesó su dinamismo y perdieron su capacidad de innovación y desarrollo de nuevos productos/servicios, fuerzas impulsoras de la economía. Sin embargo, esto no sucedía en los países capitalistas desarrollados porque, entre otras razones, sus economías estaban basadas en micro y pequeñas empresas.

Se sostiene que el socialismo fracasó por factores políticos, como la falta de libertad y la dictadura del partido único, sin embargo se debe tener en cuenta también el aspecto económico. El socialismo era un sistema económico ineficiente, ineficaz, incapaz de estar a la altura de cambios mundiales y en el cual el emprendimiento no existía. Por todo ello la economía y el modelo fracasaron. 
A igual que sucede en los países desarrollados, en Brasil la microempresa ocupa un espacio muy importante, como el que evidencian las diversas investigaciones realizadas (LEITE, 2012). Esta es la realidad del Brasil, la microempresa representa cerca del 95\% de todas las empresas (LEITE, 2012).

Desde el punto de vista de los resultados económicos, a pesar de que en la microempresa y el auto-empleo, existen evidentes aspectos negativos, como la informalidad, la baja productividad y el ingreso insuficiente, también es posible apreciar factores positivos, como el potencial de los conglomerados o clústeres.

La situación descrita es producto del espíritu empresarial popular. El Estado prácticamente no interviene en este proceso de crecimiento; hasta se puede afirmar que, por algunos momentos, está al margen del proceso. Hay que tener en consideración que en Brasil muchas microempresas son informales y no se incluyen en los escrutinios de los impuestos, sin embargo contribuyen en la generación de empleo, con los productos y/o servicios que satisfacen los deseos y las necesidades de los clientes.

En las últimas cuatro décadas, las economías más desarrolladas evolucionaron de una estructura basada en el sector de las industrias transformadoras tradicionales a un tipo de organización en donde predominan los emprendimientos de base tecnológica.

Los nuevos emprendimientos, fundamentalmente creados en forma de microempresa, se destacan básicamente por: a) la mayor flexibilidad en la implementación de la innovación; y b) la reducción de la importancia de las economías de escala.

La persistencia de elevados niveles de desempleo y de tasas de crecimiento económico ha derivado en que las autoridades económicas de todo el mundo apuestan de forma intensiva por la figura del auto-empleo y en el emprendimiento, como una forma viable de generar empleo y renta (SARAIVA e PAIVA, 2014). La presencia de emprendedores - persona que se dedica a la creación de un nuevo emprendimiento, con el objetivo de crear, mantener y fidelizar clientes - en la economía es fundamental para que surjan las microempresas.

El hecho de presenciar en una determinada economía un considerable número de microempresas no quiere decir que estemos delante de una economía emprendedora, muchos individuos creen en la empresa como alternativa al desempleo (emprendimiento de necesidad), y no por detectar una oportunidad de satisfacer un deseo o una necesidad que aún no está atendida de forma correcta, o que nadie lo tenga percibido (emprendimiento de oportunidad) (LEITE, 2012).

\subsection{Las fuerzas motrices de la economía}

Trataremos en este epígrafe el tercer punto de este trabajo: las fuerzas motrices de los cambios económicos, las cuales denotamos seguidamente: en primer lugar, la expansión del comercio mundial, "lease" globalización; en segundo lugar, las inversiones directas de los extranjeros; en tercer lugar, las innovaciones tecnológicas, siendo la más importante la innovación tecnológica; en cuarto lugar, la iniciativa empresarial o el emprendimiento y, finalmente, un ambiente propicio para que surjan y se desarrollen las empresas, asunto que es, en parte, responsabilidad de los gobiernos.

Aprovechar las oportunidades es la fuente del desarrollo humano. Por oportunidad de negocio se entiende un conjunto de circunstancias favorables asociadas a una idea de negocio que 
tenga clientes y mercado provisor. La función del emprendedor es detectar, identificar y crear oportunidades y ponerlas en práctica. El emprendimiento puede, así, entenderse como la creación y la exploración de oportunidades que muchas veces se encuentran inexploradas.

El emprendimiento es una forma de pensar, actuar y percibir el mundo. El núcleo de la cuestión del proceso emprendedor es la identificación, la creación de oportunidades de negocio que envuelve una predisposición para correr riesgos calculados.

El desarrollo de nuevas ideas de negocio se circunscriben en cuatro fuentes: la primera, la creatividad (pensar en cosas, la búsqueda de la oportunidad en la imaginación de los emprendedores) y la innovación (hacer cosas nuevas); la segunda, el emprendimiento, (el espíritu emprendedor, que organiza y combina factores de producción); la tercera, la agencia (se convierte en un agente de su propio desarrollo); y la cuarta, la sociabilidad (la capacidad de organizarse en todas las formas y niveles para alcanzar determinados objetivos).

\subsection{El emprendimiento}

Es preciso dar apoyo al egresado cuando este decide crear una empresa, para que podamos presenciar la transformación de conocimiento en riquezas. Además este apoyo debe verificarse no sólo en el momento final sino a lo largo del proceso de enseñanza Se hace necesario crear empresas, pues ellas son el fruto de los conocimientos acumulados y de experiencia durante el periodo de su formación universitaria, especialmente cuando es una microempresa, muchas veces esta es la materialización de una visión.

Para crear una empresa, se tienen que buscar experiencias relevantes y acumular conocimientos con el fin de emprender su propia aventura. El cúmulo de conocimiento es algo muy importante, consecuentemente tiene un impacto en las entidades educativas, a las cuales les corresponde asesorar y/o ayudar al alumno a adquirirla.

El emprendedor no precisa solamente de conocimientos, debe desarrollar actitudes: la capacidad de seguir adelante, de no dejarse intimidar frente a los problemas, la persistencia, el liderazgo, la creatividad, la innovación y la capacidad de convencimiento. Un emprendedor debe poder convencer a sus clientes de que su producto/servicio es bueno, a su socio de la necesidad de tomar una decisión, a sus trabajadores de la importancia de ciertas medidas, entre otros.

¿Y cómo se enseñan estas competencias que no son estrictamente conocimientos en una universidad o escuela? En la actualidad, las instituciones educativas ya incluyen en sus planes de estudio actividades orientadas a obtener estas capacidades. Muchas de estas capacidades se adquieren con la práctica y las clases facilitan su mejor aprovechamiento. (McCLELLAND, 1961)

También queremos señalar la importancia de las redes. En el emprendimiento las redes de contacto, tanto las comerciales como las personales, desempeñan un papel importante. Identificamos dos tipos de redes, las redes oficiales se denominan comerciales y las redes extraoficiales o informales se identifican como las personales.

Los emprendedores reciben ayuda y consejos que son evaluados y asimilados para luego convertidos en un factor valioso de éxito. El emprendedor busca personas capaces de ponerlos en contacto con personas clave o bien con aquellas personas que pueden ofrecer consejos e información relevante. 
Por lo tanto, se hace necesario establecer redes, debido a que las redes académicas, de conocimientos, se transforman en redes empresariales (LEITE, 2012). De ahí la importancia, nuevamente, de la experiencia previa, de conocer, por ejemplo, quien está a investigando determinado tema que interesa al emprendedor. En muchos casos, la familia de emprendedores genera más emprendedores; si el padre o madre es emprendedor, existen altas probabilidades de que el hijo también lo sea. El emprendedor puede tener la posibilidad de conocer personas que provengan de familias emprendedoras y así observar e incorporar cualidades y valores relacionados al emprendimiento. Vinculado al factor anterior esta la variable cultura. No solamente recibimos la herencia familiar y genética, que es un factor decisivo, también asimilamos las ideas y los valores de la cultura en el país en donde vivimos (HOFSTEDE, 2001). En países en donde durante épocas satanizaron a los emprendedores, no hay sitio para la innovación, para la creación de nuevas empresas, ni para la creación de nuevos productos/servicios.

Las culturas en donde se sataniza el éxito, los emprendedores son considerados como "bandidos de una película" y se rechaza todo aquello que tenga vinculación con los emprendedores. Consecuentemente nos preguntamos, ¿A dónde se puede llegar con este tipo de cultura e ideología? Es parte de la cultura emprendedora reconocer los valores de éxito y de recompensa económica, creemos que es algo legítimo sentirse orgulloso por los frutos de trabajo generados y por la capacidad personal.

La educación, que es la parte de la cultura, es también una variable fundamental. Existen países en donde se estimula la educación y el emprendimiento, y otros en donde se oferta una educación donde inculcan a las personas una mentalidad de empleado dependiente. Estas personas terminan los estudios con la expectativa de que alguien los contratará, muchos de ellos aún están a la espera de un empleador.

La universidad no se puede limitar tan sólo a dar conocimientos y enviar a sus egresados al mercado, tiene que incluir el factor del emprendimiento en la formación, de modo que sus alumnos sean capaces de crear su propio puesto de trabajo al finalizar su formación. El emprendimiento debe ser implementado en su formación desde la primera etapa de la educación de todos los individuos.

Pocas personas tienen la oportunidad de aprender la realidad de las empresas, del emprendimiento. Algunos las conocen por necesidad, generalmente individuos de pocos recursos que precisan ayudar a sus padres en alguna actividad empresarial o en una actividad informal. Sin embargo este tipo de enseñanza se puede realizar de forma organizada.

Países en donde la educación es emprendedora, sus escuelas y sus universidades desempeñan un papel importante, como proveedoras de conocimiento más especializados. Especialmente relevante para industrias y empresas de alta tecnología. Además las escuelas de negocios emprendedoras conforman una ventaja competitiva sostenible para el desarrollo de las economías de los países en donde se encuentran las instaladas (LEITE, 2012). En esta línea encontramos estudios de interés de publicación más reciente elaborados por las autoras SARAIVA e PAIVA (2014)

El factor siguiente del emprendimiento es el valor no monetario. Muchas personas se dedican a crear una empresa, no solo por la rentabilidad que el negocio ofrece, si no que puede estar fundamentada esta decisión en distintas motivaciones. El emprendedor se guía por la necesidad de auto-realización, ser su propio jefe, contribuir al desarrollo de la sociedad, el 
reconocimiento social, la independencia, entre otros. Todos estos valores deben estar presentes en la sociedad emprendedora. Una sociedad en la cual el ser independiente y ser emprendedor no es un valor explícito, no genera emprendedores. Lamentablemente existen en la actualidad ideologías que fomentan la pasividad como por ejemplo el resurgir del fenómeno del emprendimiento en Cuba.

Las personas que se basan en este último enfoque fundamentalmente prefieren un líder que les diga lo que tienen que hacer, piense por ellos y les evite preocupaciones. Si este es el valor universal en algún país, nos preguntamos ¿qué es lo que se puede esperar de él? (McCLELLAND, 1961). La libertad de emprender no es una opción entre varias posibles, representa el ápice de la autonomía.

A lo largo de este trabajo hemos desarrollado tres factores de índole personal que influyen en el emprendimiento, la experiencia, el background y la cultura. Por lo que se hace necesario ahora que analicemos el ambiente macroeconómico. No importa la dimensión de la economía, carente de recursos naturales o no, pero sí que se disponga de un sistema que favorezca el emprendimiento. Esta tendrá una enorme probabilidad de alcanzar el mismo grado de desarrollo igual que otra cualquiera que esté dotada con las mejores condiciones.

Para que los potenciales emprendedores puedan adquirir experiencia y conseguir redes de contactos, tanto comerciales como personales, se debe desarrollar en el país una actividad industrial empresarial pujante. Lo más destacable, en las economías en donde el espíritu emprendedor prevalece, es que su estructura industrial es democrática, con un gran número de microempresas, lo que incentiva el emprendimiento y genera más oportunidades. Estamos, pues, frente al ambiente económico, variables que están relacionadas con los factores anteriormente mencionados. Si en un país no existe actividad empresarial ni industrial, ¿ ¿n donde se adquiere la experiencia? ¿En donde se realizan las prácticas pre profesionales?

Buscar prácticas para sus alumnos constituye una dificultad real para las universidades. En Brasil, las grandes empresas hace un tiempo que dejaron de ofertar prácticas a estudiantes, y algunas universidades no aceptan como válidas las prácticas en microempresas por su informalidad, a pesar de que, según la investigación realizada, la experiencia en este tipo de empresas es muy importante para el emprendimiento, que la adquirida en empresas más grandes.

La experiencia que se obtiene en una empresa es tan o más valiosa que los conocimientos adquiridos en una escuela o universidad, por lo que consideramos que la incubación de empresas es una forma inteligente de obtener una experiencia emprendedora.

Criterios como estos respaldan, por ejemplo, los Programas de Incubación de Empresas, paulatinamente implementados y cuyos resultados, obteniendo buenos resultados favoreciendo el aprendizaje del emprendedor.

\subsection{La Innovación y la Contribución para Desarrollar el Espírito Emprendedor}

La innovación es la base para desarrollar el espíritu emprendedor que se rije bajo tres ejes:

I. La creación de un ambiente propicio a colaboraciones estratégicas entre universidades, institutos de investigación y empresas; 
II. Estímulo de la participación de Instituciones Científicas y Tecnológicas en el proceso de innovación; y

III. El incentivo a la innovación en la empresa.

Por otra parte cabe señalar que la innovación permite:

- Establecer alianzas estratégicas para la cooperación entre las Instituciones Científicas y Tecnológicas;

- Compartir la infraestructura y la incubación de empresas;

- Autorizar la incubación de empresas en el espacio público, compartir la infraestructura, equipamientos y recursos humanos, públicos y privados para el desarrollo tecnológico y la generación de procesos y productos;

- Facilitar la transferencia de tecnología;

- Fomentar directamente al sector productivo;

- Proporcionar la construcción de la empresa estratégica innovadora;

- Apoyar a la creación de micro y pequeñas empresas.

De todo ello se deduce que, una actividad económica de cierto volumen y el dinamismo para que surja con el emprendimiento. De otra forma será muy difícil adquirir la experiencia necesaria y obtener ventajas de redes de contactos comerciales y personales.

Reiterando, en los lugares en donde tenemos la presencia de un mayor clima empresarial y presencia de microempresas, según nuestra revisión, se apunta a una mayor manifestación del emprendimiento.

Conviene destacar una importante variable vinculada al emprendimiento que es el financiamiento. La mayoría de las personas piensa que si no dispone de financiación no puede crear empresa. Esto es falso, pues muchos de los emprendedores que estudiamos no tuvieran financiación en sus inicios; la gran mayoría utilizaron recursos propios, debido a que un banco no da crédito a un emprendedor que llegue a sus agencias solamente con ideas o esbozos de la creación de una empresa.

Muchos emprendedores consiguen recursos propios de familiares o de amigos, con ello reflejamos nuevamente a la importancia de las interrelaciones. Si no se tiene cierto grado de desarrollo industrial y si la familia no tiene ciertos recursos, difícilmente se puede crear una microempresa con posibilidades de éxito.

Lo que se observa en gran medida en Brasil son microempresas de supervivencia o de autoempleo, sin embargo no son estas las que abarcamos en nuestro estudio y sí, de las empresas con capacidad de crecimiento que requieren de cierto nivel de conocimiento basado en la experiencia y en las relaciones. El financiamiento está vinculado con las redes de contactos personales y comerciales. Los proveedores muchas veces son los que proporcionan el crédito para iniciar esta actividad, porque depositan su confianza en el emprendedor, el cual puede demostrar alguna experiencia previa.

De todo ello se deduce que la importancia de un ambiente macroeconómico que propicie y proporcione más oportunidades. En la revisión bibliográfica se detectó que en los lugares en donde las actividades emprendedoras son apoyadas e incentivadas muestran mayor crecimiento y desarrollo, la generación de empresas y de empleo también se ve favorecida (LEITE, 2012). 
También cabe señalar que, el papel del gobierno es el de generar ese ambiente macroeconómico dinámico, en donde se produzcan mayores posibilidades de creación de empresas y de nacimiento de emprendedores exitosos. Las instituciones pueden influenciar a que se tomen en consideración determinadas prácticas en función de las presiones coercitivas, miméticas o normativas (SÁNCHEZ-FERNÁNDEZ, VARGAS y REMOALDO, 2014). Esto no quiere decir que en épocas de crisis o de recensión no surjan oportunidades, también pueden ser percibidas, creadas, tal como hemos visto en diferentes casos, sin embargo el mayor peso recae en la creación de nuevas empresas, vía incubadora, y su impacto sobre la economía.

En la actualidad se percibe, entre los brasileños, una cierta predisposición a culpar de todo al gobierno, al Estado, lo cual no es la actitud constructiva. Lo mejor sería que ellos pensaran que pueden emprender y desarrollar, invertir, arriesgarse, buscar recursos. Una vez cumplidos estos ítems los emprendedores podrían plantearse solicitar un apoyo a un Estado socio, promotor. Este debe ser el papel del Estado, no el de padre protector que tiene que resolver todos los problemas del hijo.

Lamentablemente, muchas personas reclaman el paternalismo, entretanto algunos partidos y movimientos políticos lo realizan y lo aprecian, consecuentemente contribuyen poco al desarrollo del país.

Es preciso luchar por un cambio cultural, cambiar nuestras ideas y valores, también nuestras instituciones, y orientar todos nuestros esfuerzos en desarrollar el emprendimiento, que es la principal fuerza motriz del crecimiento y del desarrollo.

\section{RESULTADOS E DISCUSSÕES}

Seguidamente recogemos las siguientes conclusiones que resumimos en este decálogo:

1. El emprendedor como factor fundamental del Desarrollo Sostenible de cualquier economía (disminución de la influencia del Estado y de las grandes empresas).

2. El ser emprendedor como una actitud frente a la vida aplicable a todas las profesiones.

3. La acción de emprender aplicable a los diferentes ámbitos del ser humano: social, económico, político, cultural, artístico, entre otros (SCHUMPETER, 1988).

4. Los emprendedores y sus emprendimientos como creadores de valor y como la forma más genuina de combatir la pobreza.

5. La convicción de que se aprende a ser emprendedor (también puede ser considerada como una evidencia SARAIVA e PAIVA (2014)).

6. La necesidad de diseminar el espíritu emprendedor a través de los educadores como socios activos del aprendizaje.

7. La necesidad de incluir programas emprendedores en todos los niveles educativos (también puede ser considerada como una evidencia SARAIVA e PAIVA (2014)).

8. La metodología para aprender a enseñar a emprender debe ser en sí mismo emprendedora. 
9. Reconocimiento a la diversidad. Diversidad de culturas, de personas y de métodos para enseñar a emprender.

10. Incorporación de las mejores prácticas ya existentes en el campo del emprendimiento.

La educación emprendedora abre las puertas, esta permite a las personas desarrollar su máximo potencial, y al hacer esto, beneficia a la sociedad como conjunto. La escuela tiene una tendencia a la enseñanza tradicional, a centrarse en lo académico y enseñar materias tradicionales como matemáticas, ciencias y literatura. El programa de educación emprendedora se apoya en un enfoque complementario. Una educación completa necesita preparar a los jóvenes para el mundo del trabajo en que vivirán durante y después de sus estudios.

Los estudiantes, desde preescolar hasta la educación de nivel medio, realizan una serie de programas desarrollados para incrementar el entendimiento del mundo económico que les rodea. Enseñar desarrollando prácticas con los estudiantes interesados es asegurar que ellos mismos aprendan. No se puede aprender a ser emprendedor tan sólo leyendo un libro.

La promoción del emprendedorismo envuelve un cambio cultural basado en la premisa de que las personas pueden mejorar su calidad de vida y la de otros, tomando para sí mismos la tarea de dirigir y definir su propio destino, que impacta de dos formas: a) mejorando competencias como el liderazgo y las habilidades de comunicación; y b) a través de un incremento de la satisfacción personal, la autoestima y la motivación.

El espíritu de nuestra época es el de la globalización centrada en el emprendimiento. Las personas tienen dudas, dudan a la hora de abandonar sus miedos de emprender. Si el miedo controla nuestro comportamiento no tenemos alternativas. Consecuentemente no somos capaces de actuar porque tenemos miedo y queremos quedar libres de esa responsabilidad. El miedo es atrayente y paralizante. Sin embargo el coste de no emprender es bastante elevado. Tememos por las personas que son incapaces de emprender, estas pasan muchos años de sus vidas preocupadas por su futuro sin tomar la decisión fundamental: tener o no tener un comportamiento emprendedor.

Los individuos a veces no son conscientes del miedo de emprender que llevan dentro de sí mismos, le dan importancia a lo que "ven sus ojos", pensando en que quedarán sin los recursos invertidos, que la economía va empeorar y que debemos postergar nuestros proyectos de cambio.

El mundo no es perfecto, siempre tenemos inestabilidades. Los problemas y las dificultades siempre van a existir. Sin embargo no es verdad que el futuro será peor que el pasado. Existen miedos reales y miedos falsos. Cuando hay gran desigualdad en el mundo se produce inestabilidad. Una apuesta firme orientada al emprendimiento puede cambiar esa perspectiva, a medio y largo plazo.

Hay buenas evidencias de que el emprendimiento, una forma democrática de distribución de riquezas acumulada por los emprendedores, se fundamenta que en cualquier persona independiente de sexo, raza, religión y edad. La disputa tiene que ver con las relaciones entre los miedos de emprender más de lo que se admite. Esto quiere decir que hay que apostar por la valoración de la autoestima, pues ella es capaz de construir una cultura emprendedora, la cual se proyecta externamente al emprendedor en busca del éxito. Necesitamos recuperar la autoestima de los emprendedores brasileños, en donde podemos destacar las realizaciones de la Gerdau, fruto del desempeño de emprendedores de la visión orientada al futuro. 
El género humano se proyecta a favor de una causa noble: crear y distribuir riquezas. Por lo tanto es imprescindible que percibamos esto: entender que el emprendimiento es el fondo de todas las conquistas de la humanidad, el cual precisa ser estimulada e incentivada.

Debemos tener la conciencia del papel del espíritu emprendedor en el desarrollo de la humanidad. Cualquier estudioso del emprendimiento, de filosofía y de historia podrá observar que lo que hace que la civilización avance es el hecho de la actuación de los emprendedores, en sus correspondientes épocas, buscar siempre estar en la vanguardia de sus eras, teniendo en cuenta de sus ilimitadas capacidades de soñar. La humanidad soñaba en ser cada vez más emprendedora con el firme propósito de transformar el mundo en una perspectiva de elevada responsabilidad.

Las ideas son los escalones que los emprendedores van alcanzando, y lo que importa es que su preparación sea adecuada para enfrentarse con éxito a los desafíos que el siglo XXI les reserva. Es preciso propagar el espíritu emprendedor entre los jóvenes. El transformar ese espíritu en consciencia emprendedora capaz de modificar y transformar el mundo.

En la actualidad vivimos un momento crucial, tiempos turbulentos con cierres y aperturas de empresas a un ritmo bastante acelerado. Por lo que, el nivel de turbulencia es ponderado por el número de empresas que nacen, más de las que se mueren, de esta forma se obtiene la renovación empresarial, una medida dinámica del emprendimiento. La capacidad de emprender de una sociedad es determinante para que el tejido económico se renueve constantemente como un todo, como podemos observar en la figura 3.

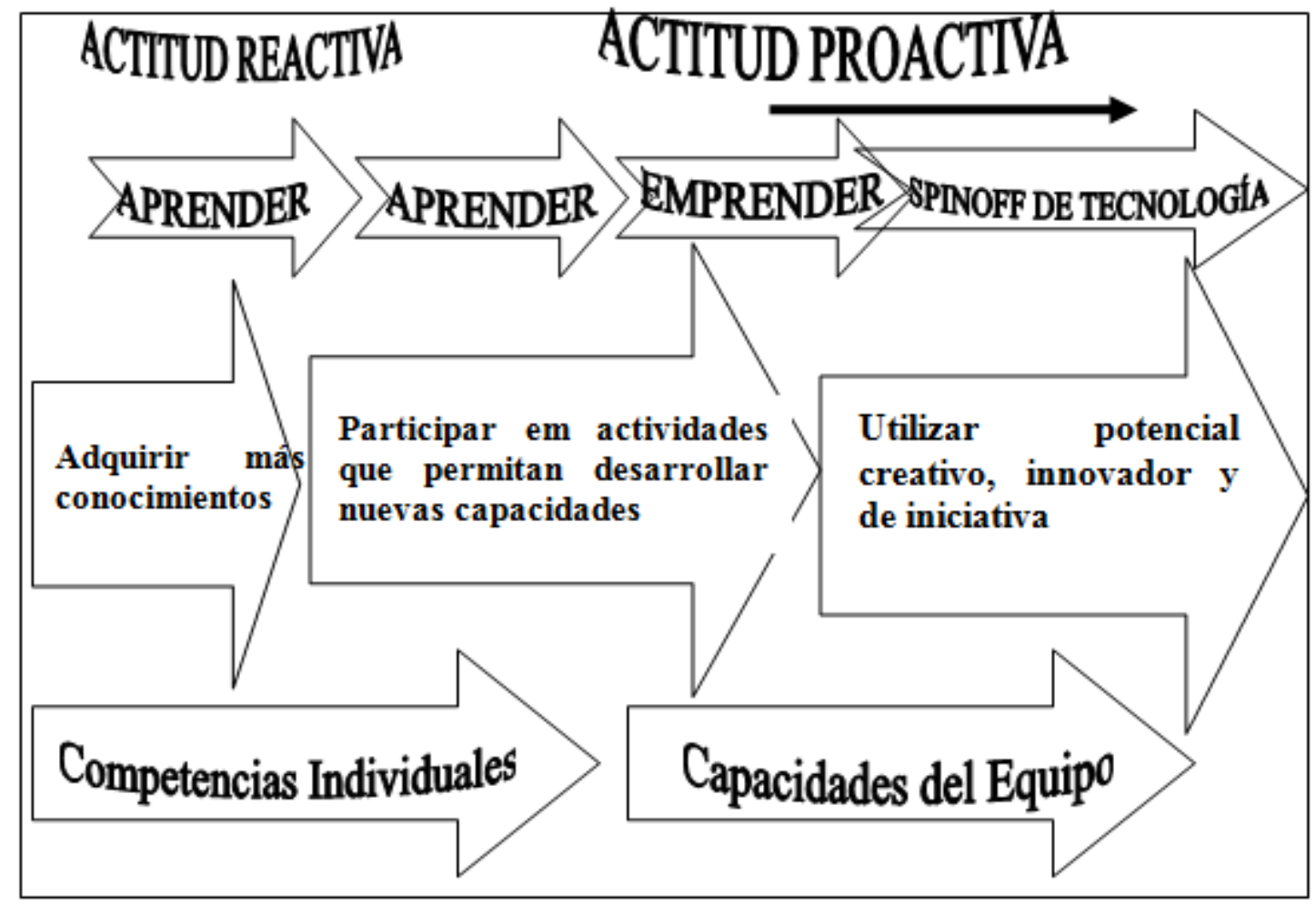

Figura 3: Emprendimiento y Actitud (LEITE, 2015)

El conocimiento y la experiencia de hacer negocios están divulgados a través de las diferentes páginas web en Internet. La profusión y la diseminación del conocimiento son fundamentales para el éxito del emprendimiento. Las experiencias emprendedoras vencedoras, 
cuando están bien divulgadas, pueden iluminar los pasos de quienes desean ser también emprendedores.

Lo que preocupa ahora a las compañías es cómo resistir a una era en la cual la capacidad de innovación está fuera de las puertas de la empresa y pueden entrar en las manos de los competidores.

Longevidad no significa nada en el mundo de la tecnología. Es necesario estar siempre en busca de nuevos caminos, exprimir la ruta de la innovación para siempre ser vanguardistas. Consecuentemente es imprescindible reinventar la empresa en todo momento (SCHUMPETER, 1984).

\section{REFERENCIAS BIBLIOGRÁFICAS}

1. CORREIA, E. B. Inovação e Periferia. Qual a relação com o desenvolvimento local?. Recife, Editora da Universidade Federal de Pernambuco - UFPE, 2012.

2. DRUCKER, P. F. Innovation and Entrepreneurship: Practices and Principles. New York: Harper \& Row, 1985.

3. HOFSTEDE, G. Culture's consequences: comparing values, behaviors, institutions, and organizations across nations, 2nd Edition. California: Sage Publications, 2001.

4. LEITE, E.F. Empreendedorismo, Inovação, Incubação de Empresas e Starups. Recife: Edições Bagaço, 2015.

5. LEITE, E.F. O Fenômeno do Empreendedorismo. São Paulo: Editora Saraiva, 2012.

6. LEITE, E.F. O processo de criação de empresas de base tecnológica via incubadora o perfil do empreendedor do Norte de Portugal e do Brasil. Universidade do Oporto. Oporto, 1999

7. MCCLELLAND, D. C. The Achieving Society. New York: D. Van Nostrand Company. p. 205-258, 1961.

8. MORGAN, K. The Exaggerated Death of Geography: Learning, Proximity and Territorial Innovation Systems. Journal of Economic Geography, 4, p. 3-21, 2004.

9. SÁNCHEZ-FERNÁNDEZ, M.D., VARGAS, A, REMOALDO, P. Institucional context and hotel social responsibility. Kybernetes, n.43, p. 413-426, 2014.

10. SÁ, A.J. de. O espaço citricultor paulista nos anos 90: A (re)afirmação de um meio técnicocientífico-informacional da globalização. Tese de Doutorado. São Paulo, FFLCH - USP, 1998.

11. SANTOS, M. Técnica, espaço, tempo: globalização e meio técnico-científico- informacional. São Paulo: Hucitec, 1994.

12.

. A Natureza do Espaço. Técnica e Tempo. Razão e Emoção. São Paulo: Nobel, 1996.

13. SARAIVA, H., PAIVA, T. Entrepreneurship Education In Portugal - Considerations On The Topic And Its Development Environment. Holos, Ano 30, v.6, p.3-15, 2014.

14. SCHUMPETER, J.A. Capitalismo, Socialismo e Democracia. Tradução Carla Santos. Rio de Janeiro: Zahar Editores, p. 98-211, 1984.

15. SCHUMPETER, J.A. Teoria do desenvolvimento econômico: uma investigação sobre lucros, capital, crédito, juro e o ciclo econômico. Tradução de Maria Sílvia Possas. São Paulo: Nova Cultura, 1988. 